\title{
Role of tenofovir disoproxil fumarate in prevention of perinatal transmission of hepatitis B virus from mother to child: a systematic review and meta-
} analysis

\author{
Young-Sun Lee ${ }^{1,{ }^{\star}}$, Ha Seok Lee ${ }^{1,}$, Ji Hoon Kim ${ }^{1}$, Sung Won Chang ${ }^{1}$, Myung Han Hyun ${ }^{2}$, Haein Bak ${ }^{1}$, \\ Sehwa Kim ${ }^{1}$, Min-jin Lee ${ }^{1}$, Chan Uk Lee ${ }^{1}$, Young Kul Jung ${ }^{1}$, Yeon Seok Seo ${ }^{1}$, Hyung Joon Yim ${ }^{1}$, \\ Jong Eun Yeon ${ }^{1}$, Soon Ho Um ${ }^{1}$, and Kwan Soo Byun ${ }^{1}$
}

Divisions of ${ }^{1}$ Gastroenterology and Hepatology and ${ }^{2}$ Oncology and Hematology, Department of Internal Medicine, Korea University Medical Center, Seoul, Korea

Received: December 27, 2018 Revised : February 21, 2019 Accepted: February 23, 2019

\section{Correspondence to}

Ji Hoon Kim, M.D.

Division of Gastroenterology and Hepatology, Department of Internal Medicine, Korea University Guro Hospital, 148 Gurodong-ro, Guro-gu, Seoul o8308, Korea

Tel: +82-2-2626-1038

Fax: +82-2-2626-3011

E-mail: kjhhepar@naver.com

*These authors contributed equally to this work.
Background/Aims: To prevent the perinatal transmission of hepatitis B virus (HBV) from mother to child, administration of an antiviral agent during pregnancy has been attempted in women who are either hepatitis B e antigen positive or have a high viral load. In this systematic review and meta-analysis with randomized controlled trials, we analyzed the efficacy and safety of tenofovir disoproxil fumarate (TDF) in preventing the perinatal transmission of $\mathrm{HBV}$ in pregnant women who have high HBV DNA titers.

Methods: Multiple comprehensive databases (PubMed, EMBASE, and Cochrane databases) were searched for studies evaluating the efficacy of TDF for the prevention of perinatal transmission of HBV.

Results: Two studies (one open label study and one double blind study) were included and analyzed. Intention-to-treat analysis (527 pregnancies) showed that the preventive effect of TDF was not significant (odds ratio [OR], 0.53; 95\% confidence interval [CI], 0.13 to $\left.2.17 ; p=0.38, I^{2}=81 \%\right)$. However, the per-protocol analysis showed that TDF significantly reduced perinatal transmission (OR, $0.10 ; 95 \%$ CI, 0.01 to $\left.0.77 ; p=0.03, I^{2}=0 \%\right)$. There was no significant difference between the TDF group and the control group with respect to maternal and fetal safety outcomes.

Conclusions: In pregnant women who have high HBV DNA titers, TDF can reduce the perinatal transmission from mother to child without significant adverse events.

Keywords: Hepatitis B virus; Tenofovir; Pregnancy; Transmission

\section{INTRODUCTION}

Chronic hepatitis $\mathrm{B}(\mathrm{CHB})$ infection is the leading cause of chronic liver disease worldwide [1], and can lead to liver cirrhosis and hepatocellular carcinoma (HCC) [2]. To achieve the World Health Organization's goal to eliminate viral hepatitis as a public health concern by 2030 [3], an increasing emphasis is placed on the importance of preventing new infections. The major route of transmission of $\mathrm{CHB}$ infection is perinatal transmission 


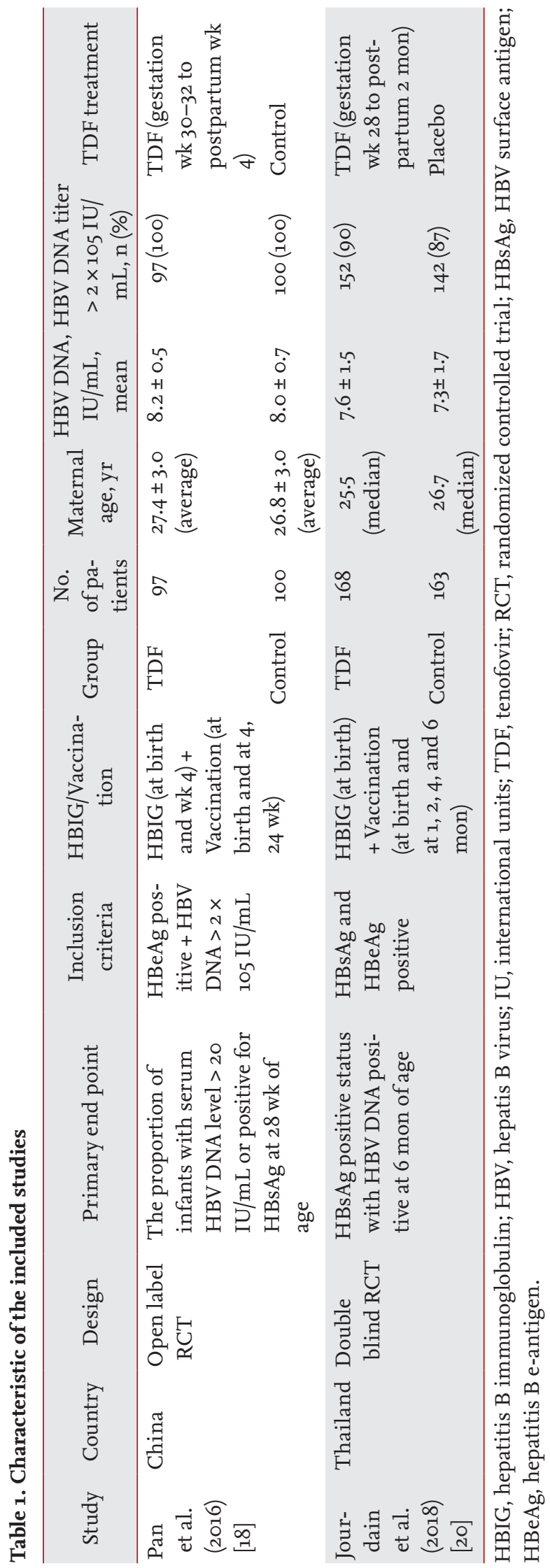

from mother to child $[4,5]$. A newborn child must receive immune prophylaxis with hepatitis B immune globulin (HBIG) and hepatitis B virus (HBV) vaccine within 12 hours after birth [6]. This immunoprophylactic strategy has reduced the rate of perinatal transmission from $90 \%$ to $10 \%$ in babies born to mothers who are hepatitis B e antigen (HBeAg) positive [7-9]. Despite immune prophylaxis, some infants are still infected by HBV from their mother, especially in cases where the mother is HBeAg positive or has a high viral load [10].

To prevent the perinatal transmission of HBV from mother to child, administration of antiviral agents like lamivudine, telbivudine, and tenofovir disoproxil fumarate (TDF) have been attempted in pregnant women who are HBeAg positive or have a high viral load [11-13]. A previous systematic review and meta-analysis concluded that lamivudine, telbivudine, and TDF significantly reduced perinatal transmission of HBV compared to immune prophylaxis alone [14]. Since lamivudine and telbivudine are no longer recommended as preferred HBV therapies due to their low antiviral efficacy and concerns regarding resistance, TDF is the preferred drug for the treatment of $\mathrm{CHB}$ and all international guidelines recommend TDF as the first line of treatment [15-17]. A randomized controlled trial (RCT) by Pan et al. [18], reported that TDF therapy with immune prophylaxis significantly reduced the perinatal transmission of $\mathrm{HBV}$ from women who are $\mathrm{HBeAg}$ positive and carrying an HBV DNA load of $>200,000 \mathrm{IU} / \mathrm{mL}$, compared to the usual management with HBIG and HBV vaccination alone. One systematic review analyzed the efficacy and safety of TDF in preventing the perinatal transmission of HBV based on 10 studies including this large-scale RCT [19]. In this study, TDF showed a significant reduction in perinatal transmission of HBV from mothers who are HBeAg positive or are carrying a high load of HBV DNA without significant adverse events. Recent international guidelines have changed their position from reservation to active recommendation of TDF therapy to mothers with a high risk of perinatal transmission when their serum HBV DNA levels are > 200,000 IU/mL [1517]. However, in a recent double blind RCT, the efficacy of TDF in preventing perinatal transmission of $\mathrm{HBV}$ was not significant compared to placebo in HBeAg-positive mothers [20]. Hence, further studies are necessary to understand the role of TDF in preventing the perina- 
tal transmission of HBV from high-risk mothers. In this systematic review and meta-analysis, we analyzed the efficacy and safety of TDF in preventing the perinatal transmission of HBV from high-risk pregnant women based on previous RCTs.

\section{METHODS}

\section{Search strategy}

This meta-analysis was performed under the guidelines and review protocols of the Preferred Reporting Items for Systematic Reviews and Meta-analysis group. Related studies were obtained and analyzed from MEDLINE (through PubMed), EMBASE, and Cochrane Library databases up until July 31, 2018. The main search keywords used included "TDF," "Hepatitis B," "pregnancy," and "perinatal transmission." Further search strategies are detailed in Supplementary Table 1.

\section{Inclusion and exclusion criteria}

Studies were included in our meta-analysis if they were: (1) RCTs, (2) conducted using TDF, (3) investigated the efficacy and safety of TDF in preventing the perinatal transmission of HBV from mother to child, and (4) published in English. We excluded studies using other antiviral agents, case reports, and retrospective studies. We also excluded studies for which only abstracts were available or that were presented only at conferences. Two authors (Y.S.L. and H.S.L.) identified the eligible studies and differences of opinion between the reviewers were resolved by discussion and consensus.

\section{Data extraction}

The same reviewers independently extracted the data including: first author; ethnicity; study design; inclusion criteria; number of participants; age; the number of infected infants at birth and at 6 months of age; and maternal and fetal adverse events such as alanine aminotransferase (ALT) elevation during pregnancy, gestational hypertension, postpartum hemorrhage, pre-term labor, fetal deformities, fetal infection, and jaundice. Perinatal transmission of HBV from mother to child was defined as the detection of the HBV surface antigen (HBsAg) or HBV DNA in infants at approximately 6 months of age.

\section{Risk of bias assessment}

The risk of bias in each study was assessed using the Cochrane risk of bias tools. Parameters considered to assess the risk of bias in each study comprised random sequence generation, allocation concealment, blinding of the participants and personnel, blinding of outcomes, incomplete outcome data, selective reporting, and other sources of bias.

\section{Statistical analysis}

All statistical analyses were performed using Review manager (RevMan) version 5.3 (The Nordic Cochrane Centre, The Cochrane Collaboration, 2014, Copenhagen, Denmark). Odds ratio (OR) with 95\% confidence intervals (CI) were analyzed for efficacy and safety profiles. The Mantel-Haenzel random-effect model was used for the analysis of inter-study heterogeneity. $I^{2}$ values of $25 \%, 50 \%$, and $75 \%$ were considered low, moderate, and high heterogeneity respectively. A $p$ value of $<0.05$ was considered statistically significant.

\section{Ethical statement}

This study is approved by by the Institutional Review

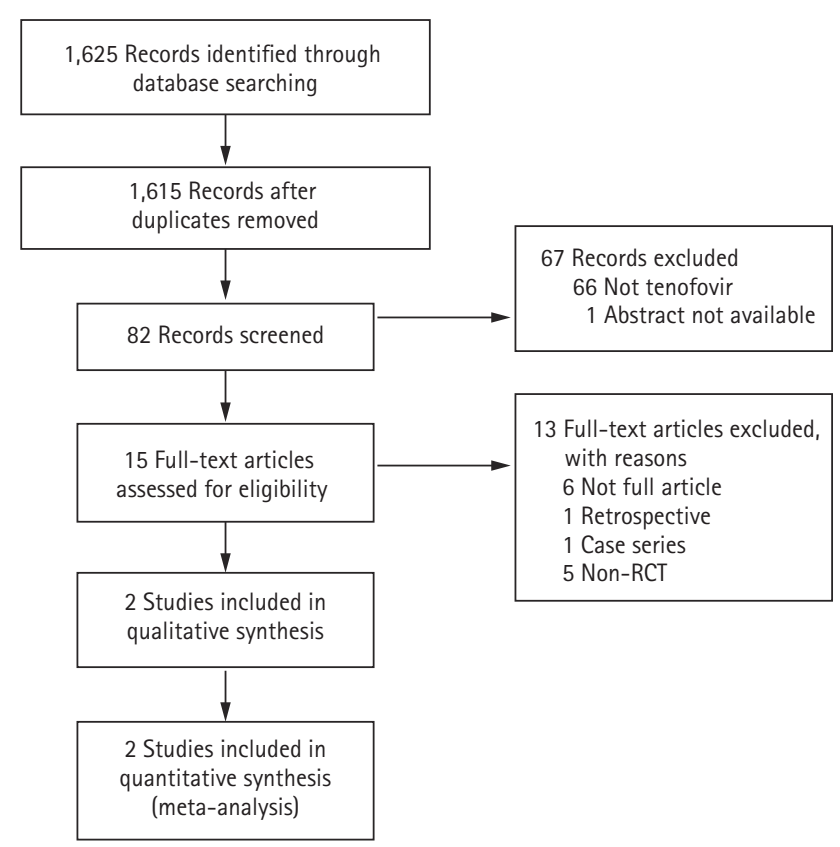

Figure 1. Flow chart of study assessment. RCT, randomized controlled trial. 
Table 2. Assessment of risk of bias

\begin{tabular}{|c|c|c|c|c|c|c|c|}
\hline Variable & $\begin{array}{c}\text { Random sequence } \\
\text { generation }\end{array}$ & $\begin{array}{c}\text { Allocation } \\
\text { concealment }\end{array}$ & $\begin{array}{l}\text { Blinding of partici- } \\
\text { pants and personnel }\end{array}$ & $\begin{array}{l}\text { Blinding of } \\
\text { outcome }\end{array}$ & $\begin{array}{c}\text { Incomplete } \\
\text { outcome data }\end{array}$ & $\begin{array}{l}\text { Selective } \\
\text { reporting }\end{array}$ & $\begin{array}{c}\text { Other source } \\
\text { of bias }\end{array}$ \\
\hline $\begin{array}{l}\text { Pan et al. } \\
(2016)[18]\end{array}$ & Low & Low & High & Unclear & Low & Low & Low \\
\hline $\begin{array}{l}\text { Jourdain et } \\
\text { al. }(2018)[20]\end{array}$ & Low & Low & Low & Unclear & Low & Low & Low \\
\hline
\end{tabular}

Intention-to-treat analysis

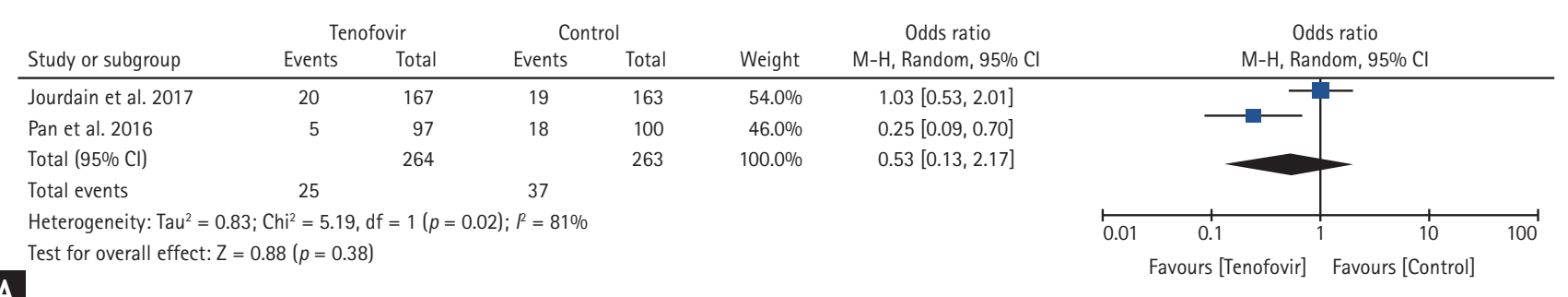

A

Per-protocol analysis

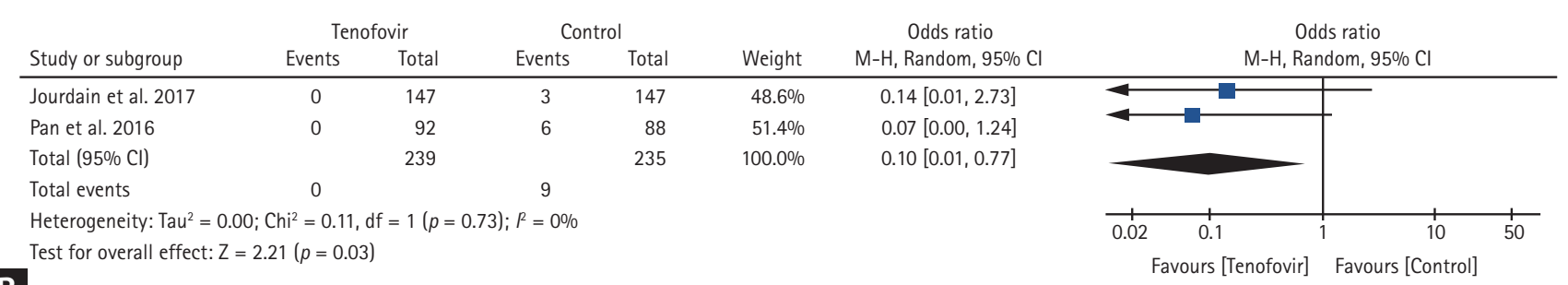

B

Figure 2. Forest plots indicating the efficacy of tenofovir disoproxil fumarate for the prevention of perinatal transmission from mother to child. (A) Intention-to-treat analysis, (B) per-protocol analysis. CI, confidence interval; M-H, Mantel-Haenszel.

Board of Korea University Guro Hospital (2019GRo443). Informed consent was waived by the board.

\section{RESULTS}

\section{Identification of the studies}

As shown in Fig. 1, 1,625 studies were searched using MEDLINE (through PubMed), EMBASE, and Cochrane Library databases. After removing any duplicated studies, the titles and abstracts of the 1,615 studies were evaluated resulting in a full review of the text articles of 15 studies conducted by two reviewers (Y.S.L. and H.S.L.). Of these 15 studies, 13 studies were excluded: full texts of six studies were not available, one was a retrospective study, one was a case series, and five studies were not RCTs. Ultimately, two studies were included in this meta-analysis.

\section{Characteristics of the studies}

Two RCTs comprising 528 pregnant women were included in this meta-analysis. The characteristics of these studies are summarized in Table 1 . The study by Pan et al. [18], was an open-label trial conducted in China, whereas the study by Jourdain et al. [20], conducted in Thailand was a double-blind RCT. The study by Pan et al. [18], included pregnant women with $\mathrm{CHB}$ who were positive for HBeAg and had a HBV DNA level of > $200,000 \mathrm{IU} / \mathrm{mL}$. On the other hand, the study by Jourdain et al. [20], included pregnant women with CHB who were positive for HBeAg; only 89.4\% (296/331) of the participants had an HBV DNA level > 200,000 IU/ mL. Infants received HBIG at birth and at week 4 , along with HBV vaccinations at birth, week 4, and week 24 in the Pan et al. [18] study. In the Jourdain et al. [20] study, infants received HBIG at birth, and 5 doses of HBV vaccine at birth, 1, 2, 4, and 6 months. In the Chinese study, 
ALT elevation

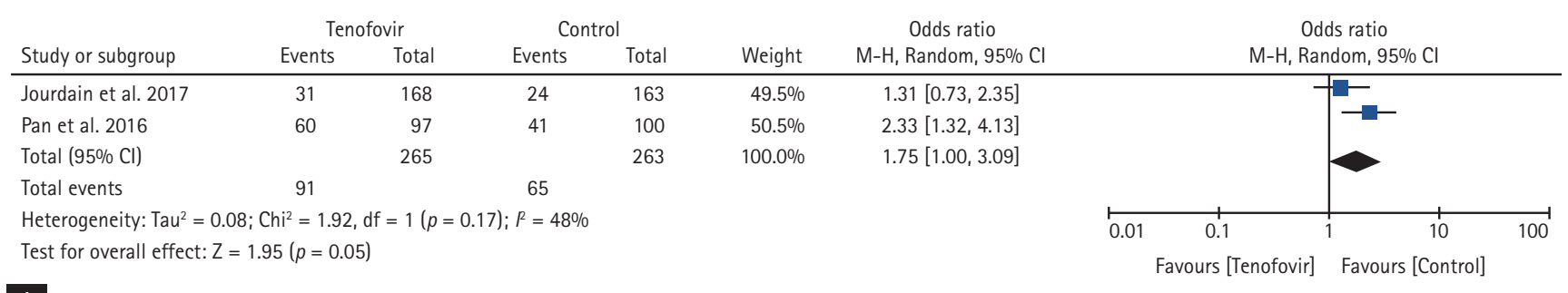

A

Gestational HTN

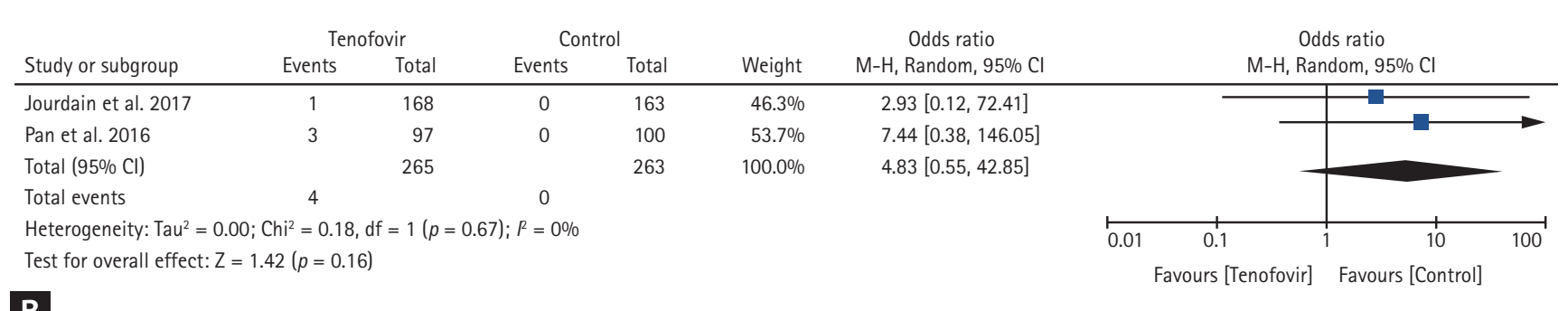

\section{B}

Postpartum hemorrhage

\begin{tabular}{|c|c|c|c|c|c|c|c|c|c|c|c|}
\hline \multirow[b]{2}{*}{ Study or subgroup } & \multicolumn{2}{|c|}{ Tenofovir } & \multicolumn{2}{|c|}{ Control } & \multirow[b]{2}{*}{ Weight } & \multirow{2}{*}{$\begin{array}{c}\text { Odds ratio } \\
\mathrm{M}-\mathrm{H}, \text { Random, } 95 \% \mathrm{Cl}\end{array}$} & \multirow{2}{*}{\multicolumn{5}{|c|}{$\begin{array}{c}\text { Odds ratio } \\
\mathrm{M}-\mathrm{H}, \text { Random, } 95 \% \mathrm{Cl}\end{array}$}} \\
\hline & Events & Total & Events & Total & & & & & & & \\
\hline Jourdain et al. 2017 & 1 & 168 & 0 & 163 & $16.3 \%$ & $2.93[0.12,72.41]$ & & & & & \\
\hline Pan et al. 2016 & 4 & 97 & 4 & 100 & $83.7 \%$ & $1.03[0.25,4.25]$ & & & & & \\
\hline Total $(95 \% \mathrm{Cl})$ & & 265 & & 263 & $100.0 \%$ & $1.22[0.34,4.46]$ & & & & & \\
\hline Total events & 5 & & 4 & & & & & & & & \\
\hline \multicolumn{7}{|c|}{ Heterogeneity: $\mathrm{Tau}^{2}=0.00 ; \mathrm{Chi}^{2}=0.34 \mathrm{df}=1(p=0.56) ;{ }^{2}=0 \%$} & 0.01 & 0.1 & 1 & 10 & 100 \\
\hline \multicolumn{7}{|c|}{ Test for overall effect: $Z=0.31(p=0.76)$} & \multicolumn{5}{|c|}{ Favours [Tenofovir] Favours [Control] } \\
\hline
\end{tabular}

C

Preterm labor

\begin{tabular}{|c|c|c|c|c|c|c|c|c|c|c|c|}
\hline \multirow{2}{*}{ Study or subgroup } & \multicolumn{2}{|c|}{ Tenofovir } & \multicolumn{2}{|c|}{ Control } & \multicolumn{3}{|c|}{ Odds ratio } & \multirow{2}{*}{\multicolumn{3}{|c|}{$\begin{array}{c}\text { Odds ratio } \\
\mathrm{M}-\mathrm{H}, \text { Random, } 95 \% \mathrm{Cl}\end{array}$}} & \\
\hline & Everits & & Evemis & I0tal & vveigni & $\mathrm{IVI- \Pi ,} \mathrm{Kantuom,} \mathrm{95 \%} \mathrm{CI}$ & & & & & \\
\hline Jourdain et al. 2017 & 2 & 168 & 9 & 163 & $58.4 \%$ & $0.21[0.04,0.97]$ & & & & & \\
\hline Pan et al. 2016 & 2 & 97 & 1 & 100 & $41.6 \%$ & $2.08[0.19,23.37]$ & & & & & \\
\hline Total $(95 \% \mathrm{Cl})$ & & 265 & & 263 & $100.0 \%$ & $0.54[0.06,5.06]$ & & & & & \\
\hline Total events & 4 & & 10 & & & & & & & & \\
\hline \multicolumn{7}{|c|}{ Heterogeneity: $\operatorname{Tau}^{2}=1.61 ; \mathrm{Chi}^{2}=2.50, \mathrm{df}=1(p=0.11) ; P^{2}=60 \%$} & 0.01 & 0.1 & 1 & 10 & $\overrightarrow{100}$ \\
\hline \multicolumn{7}{|c|}{ Test for overall effect: $Z=0.54(p=0.59)$} & \multicolumn{3}{|c|}{ Favours [Tenofovir] } & Con & \\
\hline
\end{tabular}

D

Figure 3. Forest plots for maternal safety outcomes. (A) Alanine aminotransferase (ALT) elevation. (B) Gestational hypertension (HTN). (C) Postpartum hemorrhage. (D) Pre-term labor. CI, confidence interval; M-H, Mantel-Haenszel.

TDF was administered from 30 to 32 weeks of gestation until postpartum week 4 . In the Thailand study, TDF was initiated at gestational week 28 and continued until 2 months postpartum.

\section{Quality of the included studies}

The results of the risk-of-bias analysis are shown in Table 2. Since some parameters were not mentioned in both studies, those parameters could not be evaluated. Since the Pan et al. [18] study was an open-label study, we assessed blinding as high risk. 


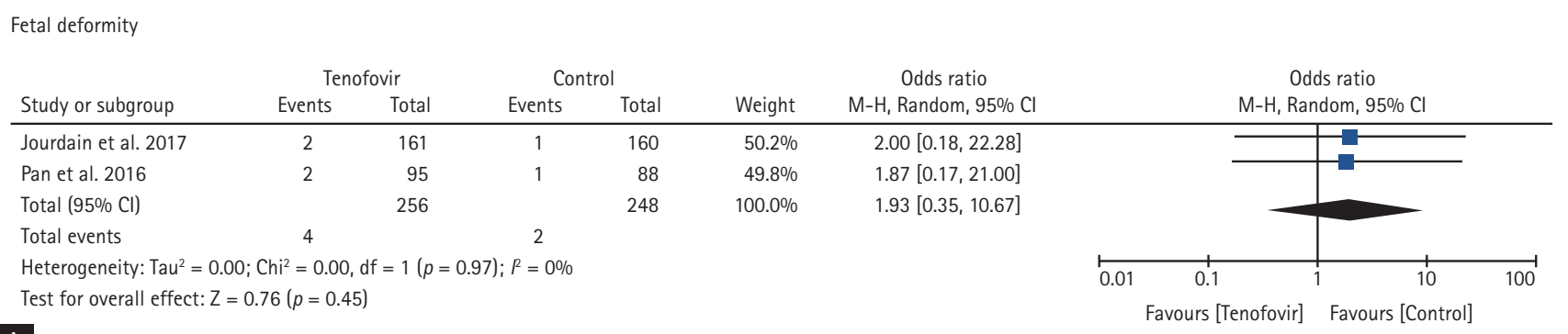

A

Fetal infection

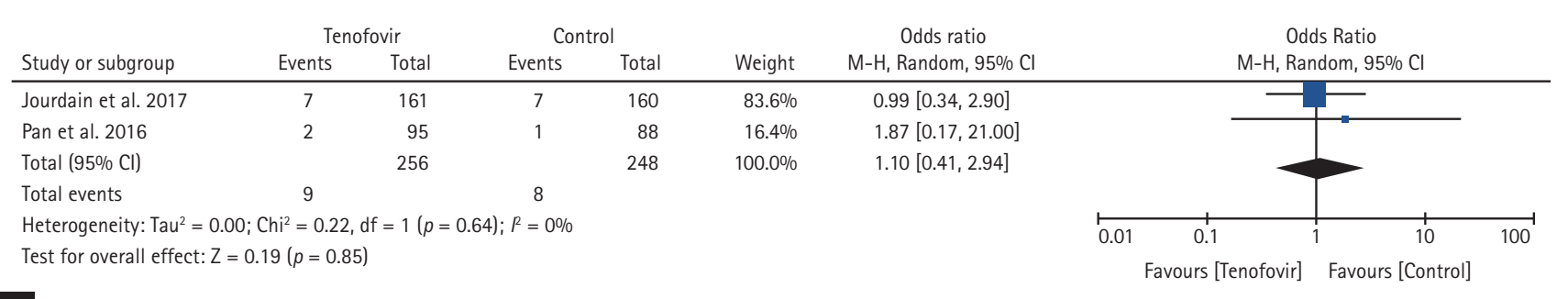

B

Postpartum hemorrhage

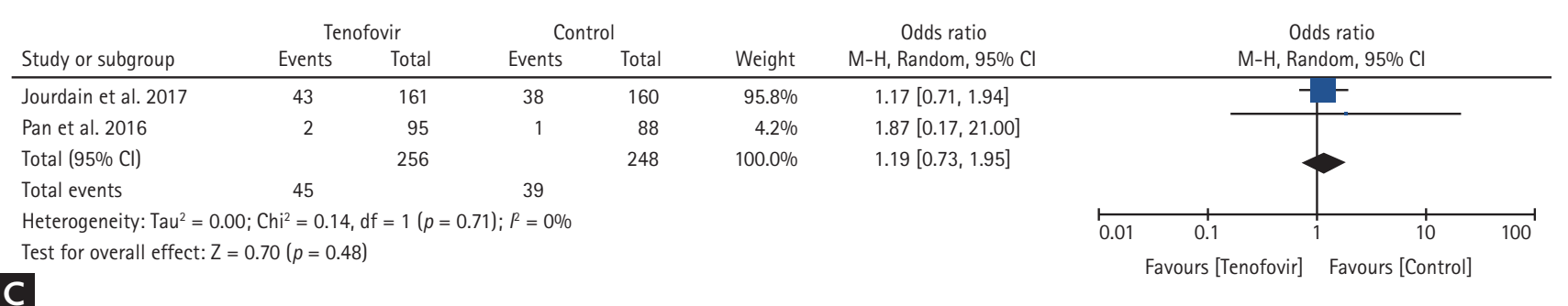

Figure 4. Forest plots for fetal safety outcomes. (A) Fetal deformity. (B) Fetal infection. (C) Fetal jaundice. CI, confidence interval; M-H, Mantel-Haenszel.

\section{Efficacy in the prevention of perinatal transmission of HBV from mother to child}

To assess the efficacy of TDF in preventing the perinatal transmission of HBV from mother to child, we checked for positivity of HBsAg in infants within 6 months of their birth, according to the intention-to-treat and per-protocol analyses. In the pooled analysis by intention-to-treat, there was no significant difference in the perinatal transmission of HBV from mother to child between the TDF and control groups (OR, 0.53; 95\% CI, 0.13 to $2.17 ; p=0.38, I^{2}=81 \%$ ) (Fig. $2 \mathrm{~A}$ ). However, the per-protocol analysis showed that TDF significantly decreased the perinatal transmission of HBV from mother to child when compared with the control group (OR, 0.10; 95\% CI, 0.01 to $0.77 ; p=0.03, I^{2}=0 \%$ ) (Fig. $2 \mathrm{~B}$ ).

\section{Maternal safety outcomes}

Maternal safety outcomes are shown in Fig. 3. Although ALT elevation throughout the study was higher in the TDF group compared to the control group, the OR was not statistically significant (OR, 1.75; 95\% CI, 1.00 to 3.09; $p=0.17, I^{2}=48 \%$ ) (Fig. 3A). Gestational hypertension development was also higher in the TDF group compared to the control group, but the OR was not significant (OR, 4.83; $95 \%$ CI, 0.55 to $42.85 ; p=0.16, I^{2}=0 \%$ ) (Fig. 3 B). The risk of postpartum hemorrhage was similar between both groups (OR, 1.22; 95\% CI, 0.34 to $4.46 ; p=0.76, I^{2}=$ ०\%) (Fig. ${ }_{3} \mathrm{C}$ ). On the other hand, TDF reduced the risk of pre-term labor, but the reduction was not significant (OR, 0.54; 95\% CI, 0.06 to 5.06; $p=0.59, I^{2}=60 \%$ ) (Fig. 3D).

\section{Fetal safety outcomes}

Fetal safety outcomes are summarized in Fig. 4. Com- 
paring the TDF and control groups, the risk of fetal deformity was slightly higher in the TDF group (OR, 1.93; 95\% CI, 0.35 to $10.67 ; p=0.45, I^{2}=0 \%$ ) (Fig. 4 A). However, it was not significant and deformities in the TDF group were relatively minor (i.e., torticollis, umbilical hernia, and talipes development). The occurrence of fetal infections (OR, 1.10; 95\% CI, 0.41 to $2.94, p=0.85, I^{2}=0 \%$ ) (Fig. $4 \mathrm{~B}$ ), and jaundice (OR, 1.19; $95 \% \mathrm{CI}, 0.73$ to $1.95 ; p=$ $\left.0.48, I^{2}=0 \%\right)$ were not significantly different between the groups (Fig. 4C).

\section{DISCUSSION}

Chronic viral hepatitis has risen from being the tenth major cause to becoming the seventh major cause of death worldwide, especially in low-income and lower-middle-income countries including east Asia, south Asia, Oceania, western sub-Saharan Africa, and central Asia [1]. Perinatal transmission of $\mathrm{HBV}$ is the major route of $\mathrm{HBV}$ infection in these endemic areas. Since the initiation of immune prophylaxis with HBIG and HBV vaccination within 12 hours after birth, the rate of perinatal transmission has been reduced from $90 \%$ to $10 \%$ in high risk mothers [16]. In a Taiwanese study, the $\mathrm{HBV}$ vaccination strategy significantly reduced the rate of progression to HCC [21]. Despite the improvement of immune prophylaxis, some infants acquired the HBV infection during the perinatal period from mothers who were either HBeAg positive or have a high HBV DNA titer. In addition to immune prophylaxis, antiviral treatment has significantly reduced the perinatal transmission [14]. However, the efficacy and safety of antiviral agents during pregnancy remains a concern. In this study, we analyzed and reviewed the efficacy and safety of TDF in preventing perinatal transmission of $\mathrm{HBV}$ from high-risk pregnant women with recent RCTs.

Antiviral therapy effectively reduced the perinatal transmission of $\mathrm{HBV}$ from $\mathrm{HBsAg}$ positive women with high viral loads (>200,000 IU/mL) to child [14]. Lamivudine was the first oral antiviral agent for the treatment of $\mathrm{CHB}$ and was effective in suppressing HBV replication and in reducing liver inflammation in both $\mathrm{HBeAg}$ positive and negative patients [22] and it was also initially used a potential antiviral agent to prevent perinatal transmission of HBV [23]. However, long-term treatment of lamivudine induced resistance and it is no longer recommended as the initial therapy for CHB [24]. Telbivudine also effectively reduced the risk of perinatal transmission of $\mathrm{HBV}$ ( $77 \%$ to $94 \%$ ) as shown in previous studies [19]. Although telbivudine is cost-effective compared to other antiviral agents, it is also not recommended as the first line treatment for CHB due to its low potency and low genetic barrier to resistance [25]. TDF was developed for the management of patients with human immunodeficiency virus (HIV) infection [26]. TDF effectively prevented HIV infection from mother to child during pregnancy [26], and it is generally considered safe $[27,28]$. Pan et al. [18], conducted the first large scale RCT to prove the preventive effect of TDF with 200 pregnant women who were $\mathrm{HBeAg}$ positive and had HBV DNA levels > 200,000 IU/mL. Although perinatal transmission of HBV from mother to child was significantly decreased, this study was an open-label trial. More recently, a study by Jourdain et al. [20] in Thailand was published with a double blind RCT setting. In this study, TDF did not show a significant reduction of perinatal transmission compared to placebo. This was probably due to a low perinatal transmission rate of $2 \%$ in the control group, which was very low compared to previous reports of $7 \%$ to $9 \%[18,29]$. The reason for low perinatal transmission of HBV in the Thailand study could be that some study subjects had low viremia having an HBV DNA load < 200,000 IU/mL (35/331, 10.6\%). In the present meta-analysis including these two RCTs that showed different results, TDF significantly reduced perinatal transmission compared to the control group in the per-protocol analysis (OR, 0.10; 95\% CI, 0.01 to $0.77 ; p=0.03, I^{2}=0 \%$ ). In most clinical studies, intention-to-treat analyses should precede per-protocol analyses to prevent an overestimation of the effect since protocol violations do not happen randomly or more frequently due to intervention associations. However, intention-to-treat analysis has an inevitable underestimation potential; therefore, most studies describe the results of the two analyses. In this study, there were conflicting results between the two analyses. However, the use of TDF for less than 6 months was the only intervention in this study and TDF is unlikely to induce clinically significant adverse effects resulting in withdrawal or loss of follow-up. In real practice, it is very important to prevent perinatal transmission of HBV from the mother 
to child because most transmitted infections will progress to CHB. Moreover, because the perinatal transmission risk of HBV from the mother to child is less than $10 \%$, even for high risk pregnancies, the anticipated bias would increase when non-compliant patients are regarded as treatment failures. Therefore, we analyzed and described both results of intention-to-treat analysis and per-protocol analysis advocating the results of the per-protocol analysis in favor of its clinical importance.

Prescribing any medication for women of childbearing age requires caution because the safety of both mother and child need to be considered. Up until June 2016, the Food and Drug Administration grouped prescription medications allowed during pregnancy under categories A, B, C, D, and X according to the risk of fetal injury. In this classification, TDF and telbivudine were categorized as B and lamivudine was categorized as C. However, the new Pregnancy and Lactation Labeling Rule has removed the pregnancy categories for drugs and has recommended indicating the individual risk of each drug [30]. The primary registry analysis from the Antiretroviral Pregnancy Registry reported that the prevalence of birth defects due to first trimester exposure to TDF is $2.32 \%$ ( 82 defects among 3,535 live births) although it cannot be concluded that malformations are due to TDF [31]. In this regard, most guidelines recommend prophylaxis with TDF in the second to third trimester $[15,16]$. A previous meta-analysis of TDF showed no significant adverse outcomes with respect to mother and child safety [19]. Similarly, in our meta-analysis, no significant concerns regarding maternal and fetal safety were noted. However, some studies of HIV-infected mothers reported concerns regarding growth retardation in infants due to the reduction in bone density by TDF [32-34], although other studies reported that perinatal exposure of TDF did not increase the risk of low birth weight or a diagnosis as small for gestational age [35,36]. Further studies are necessary to investigate the long-term influence of TDF on the growth of children born to HBV-infected mothers.

This study has several limitations. First, this meta-analysis included only two RCTs. The strength of meta-analyses increases with a rise in the number of included studies. However, two studies are sufficient to perform a meta-analysis when those two studies can be pooled and their results are similar [37]. Some meta-analyses including only two randomized controlled studies have been reported especially in rare diseases [38,39]. More RCTs are necessary to understand the effect of TDF on pregnant women. Secondly, we were unable to analyze the perinatal transmission risk of HBV from mother to child, including high viral load patients having an HBV DNA $>200,000 \mathrm{IU} / \mathrm{mL}$, because there was no detailed information available in the Thailand study [20]. Thirdly, due to the small sample size, a safety analysis did not yield meaningful results. Finally, these two studies were conducted using only an Asian population. Further studies involving various ancestries are required.

In conclusion, this meta-analysis including two large RCTs showed significant prevention of perinatal HBV transmission from mother to child. In mothers having a high-risk of transmission, TDF treatment should be considered for the prevention of perinatal HBV transmission from mother to child.

\section{KEY MESSAGE}

1. Pooled results with per-protocol analysis showed that tenofovir disoproxil fumarate (TDF) significantly decreased the perinatal transmission of hepatitis B virus (HBV) from mother to child compared to the control group.

2. There was no significant differences in the maternal and fetal safety outcomes between the TDF and control groups.

3. In HBV infected mothers with a high-risk of perinatal transmission, TDF treatment should be considered for preventing perinatal transmission of HBV from mother to child.

\section{Conflict of interest}

No potential conflict of interest relevant to this article was reported.

\section{Acknowledgments}

This study was supported by the research fund of the Korean Association for the Study of the Liver, a National Research Foundation of Korea grant from the Korean government (the Ministry of Education, Science and Technology) (2018R1A1A1A05076977), and a Korea University Grant (K1824451). 


\section{REFERENCES}

1. Stanaway JD, Flaxman AD, Naghavi M, et al. The global burden of viral hepatitis from 1990 to 2013: findings from the Global Burden of Disease Study 2013. Lancet 2016;388:1081-1088.

2. Pan CQ, Zhang JX. Natural history and clinical consequences of hepatitis B virus infection. Int J Med Sci 2005;2:36-40.

3. World Health Organization. Global Hepatitis Report, 2017. Geneva (CH): WHO, 2017 [cited 2019 Sep 29]. Available from: https:/www.who.int/hepatitis/publications/ global-hepatitis-report2017/en/.

4. Jonas MM. Hepatitis B and pregnancy: an underestimated issue. Liver Int 2009;29 Suppl 1:133-139.

5. Stevens CE, Beasley RP, Tsui J, Lee WC. Vertical transmission of hepatitis B antigen in Taiwan. N Engl J Med 1975;292:771-774.

6. Mast EE, Weinbaum CM, Fiore AE, et al. A comprehensive immunization strategy to eliminate transmission of hepatitis B virus infection in the United States: recommendations of the Advisory Committee on Immunization Practices (ACIP) Part II: immunization of adults. MMWR Recomm Rep 2006;55:1-33.

7. Chang MH, Chen CJ, Lai MS, et al. Universal hepatitis B vaccination in Taiwan and the incidence of hepatocellular carcinoma in children. Taiwan Childhood Hepatoma Study Group. N Engl J Med 1997;336:1855-1859.

8. Lee C, Gong Y, Brok J, Boxall EH, Gluud C. Hepatitis B immunisation for newborn infants of hepatitis B surface antigen-positive mothers. Cochrane Database Syst Rev 2006;2:CDoo4790.

9. Ni YH, Huang LM, Chang MH, et al. Two decades of universal hepatitis B vaccination in taiwan: impact and implication for future strategies. Gastroenterology 2007;132:1287-1293.

10. Zou H, Chen Y, Duan Z, Zhang H, Pan C. Virologic factors associated with failure to passive-active immunoprophylaxis in infants born to HBsAg-positive mothers. J Viral Hepat 2012;19:e18-e25.

11. Xu WM, Cui YT, Wang L, et al. Lamivudine in late pregnancy to prevent perinatal transmission of hepatitis $B$ virus infection: a multicentre, randomized, double-blind, placebo-controlled study. J Viral Hepat 2009;16:94-103.

12. Han GR, Cao MK, Zhao W, et al. A prospective and open-label study for the efficacy and safety of telbivudine in pregnancy for the prevention of perinatal transmission of hepatitis B virus infection. J Hepatol 2011;55:12151221.

13. Chen HL, Lee CN, Chang $\mathrm{CH}$, et al. Efficacy of maternal tenofovir disoproxil fumarate in interrupting mother-to-infant transmission of hepatitis B virus. Hepatology 2015;62:375-386.

14. Brown RS Jr, McMahon BJ, Lok AS, et al. Antiviral therapy in chronic hepatitis B viral infection during pregnancy: a systematic review and meta-analysis. Hepatology 2016;63:319-333.

15. Terrault NA, Lok ASF, McMahon BJ, et al. Update on prevention, diagnosis, and treatment of chronic hepatitis B: AASLD 2018 hepatitis B guidance. Hepatology 2018;67:1560-1599.

16. European Association for the Study of the Liver. EASL 2017 Clinical Practice Guidelines on the management of hepatitis B virus infection. J Hepatol 2017;67:370-398.

17. Korean Association for the Study of the Liver. KASL clinical practice guidelines: management of chronic hepatitis B. Clin Mol Hepatol 2016;22:18-75.

18. Pan CQ, Duan Z, Dai E, et al. Tenofovir to prevent hepatitis $\mathrm{B}$ transmission in mothers with high viral load. $\mathrm{N}$ Engl J Med 2016;374:2324-2334.

19. Hyun $\mathrm{MH}$, Lee YS, Kim JH, et al. Systematic review with meta-analysis: the efficacy and safety of tenofovir to prevent mother-to-child transmission of hepatitis B virus. Aliment Pharmacol Ther 2017;45:1493-1505.

20. Jourdain G, Ngo-Giang-Huong N, Harrison L, et al. Tenofovir versus placebo to prevent perinatal transmission of hepatitis B. N Engl J Med 2018;378:911-923.

21. Chang MH, You SL, Chen CJ, et al. Long-term effects of hepatitis B immunization of infants in preventing liver cancer. Gastroenterology 2016;151:472-480.

22. Lai CL, Chien RN, Leung NW, et al. A one-year trial of lamivudine for chronic hepatitis B. Asia Hepatitis Lamivudine Study Group. N Engl J Med 1998;339:61-68.

23. Li XM, Yang YB, Hou HY, et al. Interruption of HBV intrauterine transmission: a clinical study. World J Gastroenterol 2003;9:1501-1503.

24. Fischer KP, Gutfreund KS, Tyrrell DL. Lamivudine resistance in hepatitis B: mechanisms and clinical implications. Drug Resist Updat 2001;4:118-128.

25. Wang W, Wang J, Dang S, Zhuang G. Cost-effectiveness of antiviral therapy during late pregnancy to prevent perinatal transmission of hepatitis B virus. PeerJ 2016;4:e1709. 
26. Gallant JE, Staszewski S, Pozniak AL, et al. Efficacy and safety of tenofovir DF vs stavudine in combination therapy in antiretroviral-naive patients: a 3-year randomized trial. JAMA 2004;292:191-201.

27. Pintye J, Baeten JM, Celum C, et al. Maternal tenofovir disoproxil fumarate use during pregnancy is not associated with adverse perinatal outcomes among HIV-infected east African women: a prospective study. J Infect Dis 2017;216:1561-1568.

28. Wang L, Kourtis AP, Ellington S, Legardy-Williams J, Bulterys M. Safety of tenofovir during pregnancy for the mother and fetus: a systematic review. Clin Infect Dis 2013;57:1773-1781.

29. Chen HL, Lin LH, Hu FC, et al. Effects of maternal screening and universal immunization to prevent mother-to-infant transmission of HBV. Gastroenterology 2012;142:773-781.e2.

30. Pernia S, DeMaagd G. The new pregnancy and lactation labeling rule. $\mathrm{P}$ T 2016;41:713-715.

31. Antiretroviral Pregnancy Registry Steering Committee. Antiretroviral pregnancy registry interim report [Internet]. Wilmington (NC): Registry Coordinating Center, 2019 [cited 2019 Sep 29]. Available from: http://apregistry. com/forms/exec-summary.pdf.

32. Siberry GK, Jacobson DL, Kalkwarf HJ, et al. Lower newborn bone mineral content associated with maternal use of tenofovir disoproxil fumarate during pregnancy. Clin Infect Dis 2015;61:996-1003.
33. Stellbrink HJ, Orkin C, Arribas JR, et al. Comparison of changes in bone density and turnover with abacavir-lamivudine versus tenofovir-emtricitabine in HIV-infected adults: 48-week results from the ASSERT study. Clin Infect Dis 2010;51:963-972.

34. Mofenson LM, Baggaley RC, Mameletzis I. Tenofovir disoproxil fumarate safety for women and their infants during pregnancy and breastfeeding. AIDS 2017;31:213232.

35. Ransom CE, Huo Y, Patel K, et al. Infant growth outcomes after maternal tenofovir disoproxil fumarate use during pregnancy. J Acquir Immune Defic Syndr 2013;64:374-381.

36. Siberry GK, Williams PL, Mendez H, et al. Safety of tenofovir use during pregnancy: early growth outcomes in HIV-exposed uninfected infants. AIDS 2012;26:1151-1159.

37. Ryan R; Cochrane Consumers and Communication Review Group. Cochrane Consumers and Communication Group: meta-analysis [Internet]. London (UK): Cochrane Consumers and Communication, 2016 [cited 2019 Sep 29]. Available from: http://cccrg.cochrane.org.

38. Crins ND, Rover C, Goralczyk AD, Friede T. Interleukin-2 receptor antagonists for pediatric liver transplant recipients: a systematic review and meta-analysis of controlled studies. Pediatr Transplant 2014;18:839-850.

39. Miller RG, Mitchell JD, Moore DH. Riluzole for amyotrophic lateral sclerosis (ALS)/motor neuron disease (MND). Cochrane Database Syst Rev 2012;3:CDoo1447. 


\section{Supplementary Table 1. Search strategy}

\begin{tabular}{|c|c|}
\hline Database & Search keywords \\
\hline PubMed & 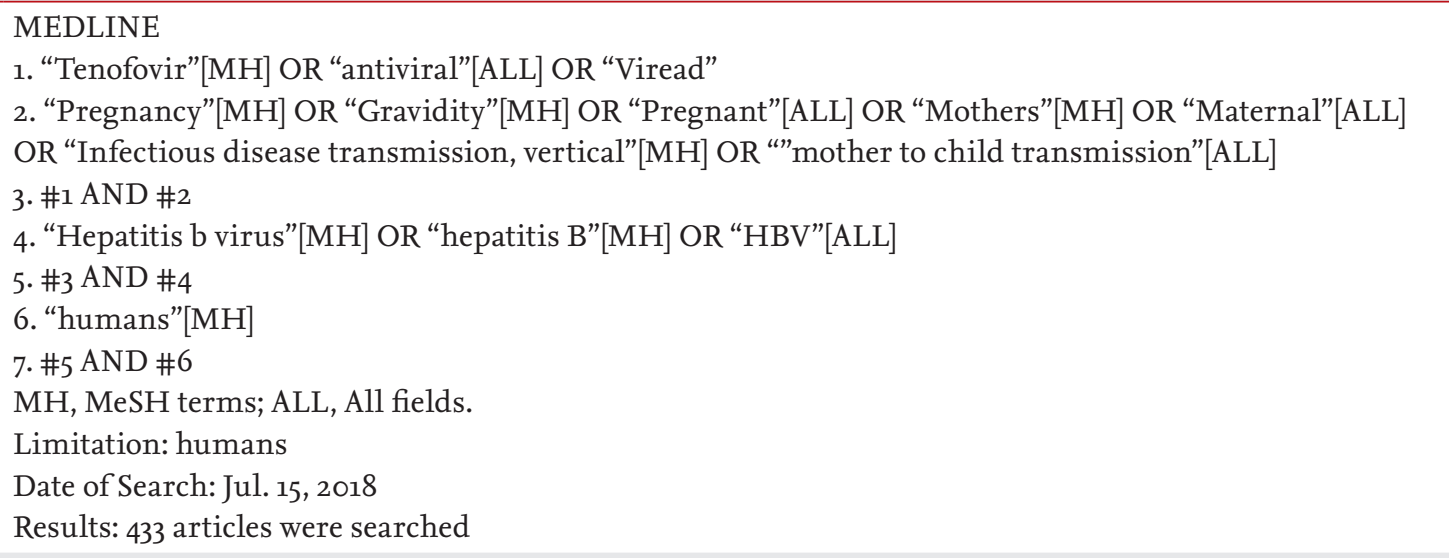 \\
\hline EMBASE & $\begin{array}{l}\text { EMBASE } \\
\text { 1. 'Tenofovir'/exp OR 'antiviral agent'/exp OR 'viread'/exp } \\
\text { 2. 'Pregnant woman'/exp OR 'pregnancy'/exp or 'adult to child transmission' OR 'mother to child trans- } \\
\text { mission'/exp OR 'Vertical transmission'/exp } \\
\text { 3. \#1 AND \#2 } \\
\text { 4. 'Chronic hepatitis B'/exp OR 'hepatitis B virus' OR 'HBV'/exp OR ‘CHB' } \\
\text { 5. \#3 AND \#4 } \\
\text { 6. \#5 AND [humans]/lim } \\
\text { Exp, explosion search; lim, limitation. } \\
\text { Limitation: humans } \\
\text { Date of Search: Jul. } 15 \cdot 2018 \\
\text { Results: } 1,125 \text { articles were searched }\end{array}$ \\
\hline Cochrane & $\begin{array}{l}\text { The Cochrane Library } \\
\text { 1. (Tenofovir) OR (Viread) OR (Antiviral agent) } \\
\text { 2. (Pregnant woman) OR (pregnancy) OR (gravidity) OR (mother to child transmission) OR (Vertical trans- } \\
\text { mission) } \\
\text { 3. \#1 AND \#2 } \\
\text { 4. (Hepatitis B) OR (Chronic hepatitis B) OR (HBV) } \\
\text { 5. \#3 AND \#4 } \\
\text { All field text searched. } \\
\text { Date of Search: Jul. 15, } 2018 \\
67 \text { Articles were searched. }\end{array}$ \\
\hline
\end{tabular}

